\title{
Observation of interspecies Feshbach resonances in an ultracold Rb-Cs mixture
}

\author{
K. Pilch ${ }^{1}$, A. D. Lange ${ }^{1}$, A. Prantner ${ }^{1}$, G. Kerner ${ }^{2}$, F. Ferlaino ${ }^{1 *}$, H.-C. Nägerl ${ }^{1}$, R. Grimm ${ }^{1,2}$ \\ ${ }^{1}$ Institut für Experimentalphysik und Zentrum für Quantenphysik, \\ Universität Innsbruck, 6020 Innsbruck, Austria \\ ${ }^{2}$ Institut für Quantenoptik und Quanteninformation, \\ Österreichische Akademie der Wissenschaften, \\ 6020 Innsbruck, Austria \\ (Dated: November 5, 2018)
}

\begin{abstract}
We report on the observation of interspecies Feshbach resonances in an ultracold, optically trapped mixture of $\mathrm{Rb}$ and $\mathrm{Cs}$ atoms. In a magnetic field range up to $300 \mathrm{G}$ we find 23 interspecies Feshbach resonances in the lowest spin channel and 2 resonances in a higher channel of the mixture. The extraordinarily rich Feshbach spectrum suggests the importance of different partial waves in both the open and closed channels of the scattering problem along with higher-order coupling mechanisms. Our results provide, on one hand, fundamental experimental input to characterize the $\mathrm{Rb}$-Cs scattering properties and, on the other hand, identify possible starting points for the association of ultracold heteronuclear RbCs molecules.
\end{abstract}

PACS numbers: $34.50 .-\mathrm{s}, 67.60 . \mathrm{Bc}, 34.50 . \mathrm{Cx}, 67.40 . \mathrm{Hf}$

\section{INTRODUCTION}

Over the last years ultracold atomic physics has entered the new terrain of more complex and composite quantum systems, such as quantum-degenerate atomic mixtures and molecular quantum gases. Great efforts have been made to produce heteronuclear mixtures with several combinations of atomic species [1, 2, 3, 4, 5] and isotopes [6, 7, 8, 9]. A rich variety of phenomena can be addressed with such mixtures as a result of different interactions, masses, and optical and magnetic properties. Moreover, two-species mixtures are very attractive systems for molecular physics [10, 11, 12]. A particular interest consists in heteronuclear molecules in the rovibrational ground state as they carry a large permanent electric dipole moment, allowing to produce strongly interacting dipolar quantum gases [13].

Rubidium and cesium, the two heaviest stable alkali species, are both well established in Bose-Einstein condensate (BEC) experiments. While Rb represents the first atomic species ever condensed [14], cesium BEC had to await the development of efficient optical trapping methods [15, 16]. The individual two-body interaction properties of $\mathrm{Rb}$ and $\mathrm{Cs}$ are very well understood as a result of extensive studies by Feshbach spectroscopy [17, 18, 19]. Cesium shows a quite unique scattering behavior because of a very large background scattering length in combination with many Feshbach resonances and pronounced high-order Feshbach coupling. A few experiments on $\mathrm{Rb}-\mathrm{Cs}$ mixtures have been performed in magnetic traps [20, 21, 22]. The observation of rapid thermalization between the two different species [20, 21] points to a large interspecies background scattering length [23]. Feshbach resonances in the Rb-Cs system have not been observed so far. Knowledge on such resonances is desired both as spectroscopic input to precisely determine the scattering properties of the mixture and to enable experimental control of the inter-

${ }^{*}$ Corresponding author; francesca.ferlaino@uibk.ac.at species interaction.

A special motivation for combining $\mathrm{Rb}$ and $\mathrm{Cs}$ is the production of a quantum gas of polar molecules, as recently demonstrated for a Bose-Fermi mixture of bosonic $\mathrm{Rb}$ and fermionic $\mathrm{K}$ atoms [12]. As the Rb-Cs mixture is a Bose-Bose system, the molecules would be bosons and could eventually form a condensate of polar molecules [13]. For studying dipolar phenomena in such a system, RbCs molecules in the rovibrational ground state are expected to carry a comparatively large electric dipole moment of 1.25 debye [24]. For this application, Feshbach resonances have proven an efficient tool to associate molecules and thus to serve as a gateway from the atomic into the molecular world [17, 25]. The Feshbach molecules are then transferred by optical Raman processes into the rovibrational ground state [12, 26, 27].

In this Article, we report on Feshbach spectroscopy in an optically trapped mixture of ${ }^{87} \mathrm{Rb}$ and ${ }^{133} \mathrm{Cs}$ atoms. After several optical cooling stages, our ultracold mixture is loaded into an optical dipole trap (Sec. II). We perform the Feshbach spectroscopy in two different spin state combinations (Sec. III), exploring a magnetic field range of up to $300 \mathrm{G}$. We find a total of 23 interspecies resonances for $\mathrm{Rb}$ and $\mathrm{Cs}$ atoms in their lowest spin states. The extraordinarily rich spectrum indicates that dipolar interactions, such as the second-order spin-orbit interaction, play an important role for the scattering properties of the mixture (Sec. IV). Finally, we discuss prospects to associate heteronuclear Feshbach molecules (Sec. V).

\section{PREPARATION OF AN OPTICALLY TRAPPED Rb-Cs MIXTURE}

The preparation of an ultracold optically trapped $\mathrm{Rb}-\mathrm{Cs}$ mixture employs several stages of cooling and trapping acting simultaneously on both species. The $\mathrm{Rb}$ and $\mathrm{Cs}$ atoms are first loaded into a two-color magneto-optical trap (MOT) from a Zeeman slowed atomic beam. The mixture is further cooled by applying two-color degenerate Raman sideband cooling (DRSC), which also polarizes the atoms into 
their lowest spin state. The sample is then loaded into a levitated optical dipole trap, which is used to probe heteronuclear Feshbach resonances.

\section{A. Laser cooling}

The $\mathrm{Rb}$ and $\mathrm{Cs}$ atoms are initially emitted from a doublespecies oven operating at a temperature of $100^{\circ} \mathrm{C}$ and $80^{\circ} \mathrm{C}$ for $\mathrm{Rb}$ and $\mathrm{Cs}$, respectively [28]. The average longitudinal velocity of $\mathrm{Rb}(\mathrm{Cs})$ is about $260(210) \mathrm{m} / \mathrm{s}$. The two-species atomic beam is slowed down by using the Zeeman slowing technique. The Zeeman-slower field is optimized for Cs, but it works well also for $\mathrm{Rb}$ because of the similar properties of the two species. The decelerated atoms are then collected into a two-color MOT, simultaneously confining and cooling the two species. The MOTs operate in a standard way, i.e. for $\mathrm{Rb}$ on the $5 S_{1 / 2}(F=2) \rightarrow 5 P_{3 / 2}\left(F^{\prime}=3\right)$ atomic transition and for $C$ s on the $6 S_{1 / 2}(F=4) \rightarrow 6 P_{3 / 2}\left(F^{\prime}=5\right)$ transition. The MOT beams for $\mathrm{Rb}$ and $\mathrm{Cs}$ are first superimposed on dichroic mirrors and then sent through a single-mode optical fiber to ensure optimum spatial overlap of both field components. We use broadband polarization optics suitable for both the $\mathrm{Rb}$ and the Cs wavelengths. After 10 s of MOT loading, we switch off the Zeeman-slower light and we apply a compression stage for $15 \mathrm{~ms}$. This is performed by ramping up the magnetic gradient field used for the MOT by a factor of two, up to a maximum gradient of $14 \mathrm{G} / \mathrm{cm}$ along the symmetry axis, and we simultaneously detune the $\mathrm{Rb}(\mathrm{Cs})$ MOT light from about -10 (-8) $\mathrm{MHz}$ to -110 (-80) MHz. At the end of the compression phase, we switch off the MOT beams and the magnetic gradient field.

The loading efficiency of a two-species MOT can be strongly affected by inelastic interspecies collisions. For the $\mathrm{Rb}-\mathrm{Cs}$ combination strong loss caused by interspecies lightassisted collisions has been reported in Ref. [22]. Such losses crucially depend on the spatial overlap between the species and can be suppressed by introducing a relative displacement. However a good spatial overlap is required to finally load the mixture into the optical dipole trap.

To meet both requirements, we make use of a spatial displacement of the trapped cloud induced by the radiation pressure of the Zeeman-slowing field and controlled by finetuning its frequency. Subsequently, the two atomic clouds overlap during the compression stage, in which the Zeeman field is off.

For optimized loading of both MOTs, we measure about $5 \times 10^{8}$ atoms for each species at a temperature of about $40 \mu \mathrm{K}$. For some experiments (Sec. II.B) it is desirable to introduce an imbalance between the atom number of the two species. This can be done by selectively reducing the power of the respective MOT-light component.

We further cool the compressed mixture by applying a twocolor version of DRSC. This technique has the advantage to provide lower temperatures and higher phase-space densities as compared to simple molasses cooling. Furthermore DRSC polarizes the two species in their respective lowest spin states, labeled as $\mathrm{Rb}|1,1\rangle$ for $\mathrm{Rb}$ and $\mathrm{Cs}|3,3\rangle$ for $\mathrm{Cs}$, where the first number indicates the total spin quantum number $F$ and the

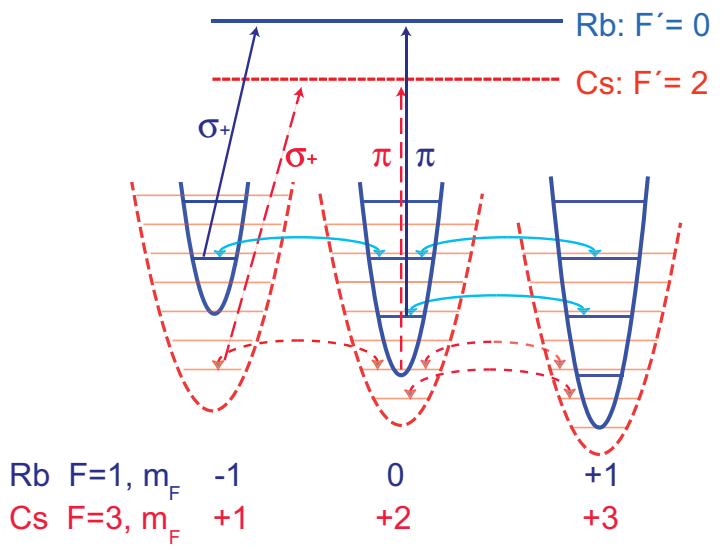

FIG. 1: color online. Illustration of the two-color DRSC technique applied simultaneously to the $\mathrm{Rb}$ (blue solid lines) and $\mathrm{Cs}$ (red dashed lines) atoms. For each species, a cycling transition consists of a two-photon Raman transition (indicated by the curved arrows) between vibrational levels of $m_{F}$-dependent harmonic micro-traps followed by optical pumping to the lowest Zeeman sublevel (not shown). $\mathrm{Rb}$ and $\mathrm{Cs}$ atoms accumulate in the vibrational ground state of the $m_{F}=1$ and $m_{F}=3$ levels, respectively, which, as dark states, are essentially decoupled from the light field.

second its projection $m_{F}$ along the magnetic field axis. In single-species experiments, DRSC has been proven a powerful method to cool and spin polarize individually both $\mathrm{Cs}$ [29, 30] and $\mathrm{Rb}$ atoms [31]. Here we extend this technique to the case of an atomic mixture. The basic concept of this cooling scheme adapted for double-species operation is illustrated in Fig. 1. The blue solid lines refer to the $\mathrm{Rb}$ and the red dashed lines to the $\mathrm{Cs}$ case. The $\mathrm{Rb}$ and $\mathrm{Cs}$ atoms are simultaneously captured in two independent three-dimensional optical lattices. The lattice light for the two species is overlapped using dichroic mirrors. We produce the lattice using a four-beam configuration [16, 32], with total power of about $28 \mathrm{~mW}$ and $20 \mathrm{~mW}$ for $\mathrm{Rb}$ and $\mathrm{Cs}$, respectively. The waist of each beam is about $1 \mathrm{~mm}$. For Cs atoms in the lowest hyperfine manifold $(\mathrm{F}=3)$, the lattice light is red-detuned by 9.2 $\mathrm{GHz}$ from the $6 S_{1 / 2}(F=3) \rightarrow 6 P_{3 / 2}\left(F^{\prime}=2\right)$ transition [33]. The $\mathrm{Rb}$ lattice is red-detuned by about $18 \mathrm{GHz}$ with respect to the $5 S_{1 / 2}(F=1) \rightarrow 5 P_{3 / 2}\left(F^{\prime}=0\right)$ transition. We observe that the lattice light for one species has negligible effect on the other species. The lattices provide two independent arrays of harmonic microtraps.

We apply a small homogenous magnetic field to Zeeman shift the harmonic microtraps in such a way that the $\left(m_{F}, v\right)$ state is nearly degenerate with the $\left(m_{F}+1, v+1\right)$ state, where $v$ refers to vibrational quantum number. Once this condition is reached, the lattice light couples states with $\left(m_{F}, v\right)$ and $\left(m_{F}+1, v+1\right)$ via a two-photon Raman transition. Typical magnetic field values are about $500 \mathrm{mG}$. To fulfill simultaneously the degeneracy condition for both $\mathrm{Rb}$ and $\mathrm{Cs}$ atoms, we first optimize on the sample of Cs atoms and we then appropriately tune the power of the Rb lattice.

Two polarizing laser beams are applied to optical pump the two species into their lowest spin states, $\mathrm{Rb}|1,1\rangle$ and 
Cs $|3,3\rangle$. The optical pumping fields are resonant with the $F=1 \rightarrow F^{\prime}=0$ and the $F=3 \rightarrow F^{\prime}=2$ transitions for $\mathrm{Rb}$ and Cs, respectively. A mixed $\sigma^{+} / \pi$ polarization of this light is necessary to assure that the atoms are driven towards the dark states $\left(m_{F}=3, v=0\right)$ for $\mathrm{Cs}$ and $\left(m_{F}=1, v=0\right)$ for $\mathrm{Rb}$. After $10 \mathrm{~ms}$ of DRSC, we ramp down adiabatically the lattice power. For maximum loading of both species, we typically measure $10^{8}$ atoms for each species at temperatures of about $3 \mu \mathrm{K}$ and $2 \mu \mathrm{K}$ for $\mathrm{Rb}$ and $\mathrm{Cs}$, respectively.

\section{B. Preparation in optical dipole trap}

After the two-color DRSC stage, we load the atoms into a large-volume levitated dipole trap [34]. Our choice of this trap configuration is motivated by two main reasons. First, the trap acts as a perfect spin filter as the levitation condition is only fulfilled for a specific magnetic substate. Therefore our mixture is free of spin impurities and corresponding ambiguities in spin channels of observed Feshbach resonances. Second, such a trap setup has the advantage to cancel the influence of the gravitational force using magnetic levitation. It is routinely employed in several experiments to produce a BEC of Cs atoms [15, 16, 35, 36] and therefore holds prospects for future experiments on two-species BECs of $\mathrm{Rb}$ and $\mathrm{Cs}$.

In our experiment, the optical field is generated by an ytterbium fiber laser (IPG YLR-100) with a central wavelength of about $1070 \mathrm{~nm}$ and a linewidth of about $3 \mathrm{~nm}$. The dipole trap is produced by two horizontally propagating laser beams crossing in the horizontal plane at a nearly right angle [37]. Each beam has a power of about $23 \mathrm{~W}$ and a waist of about $500 \mu \mathrm{m}$. The $\mathrm{Rb}$ atoms experience a confining potential that is a factor of 1.7 shallower than the one for Cs. This is due to the larger detuning and the lower optical polarizability of Rb. For our typical parameters, the trap depth for $\mathrm{Rb}(\mathrm{Cs})$ atoms is $12(20) \mu \mathrm{K}$.

A magnetic field gradient of $31.2 \mathrm{G} / \mathrm{cm}$ is applied along the vertical direction to hold the atoms against gravity, similarly to Ref. [15]. The levitation condition is simultaneously fulfilled for both $\mathrm{Rb}$ and $\mathrm{Cs}$ atoms in their absolute ground states as a result of a fortuitous coincidence in the magnetic-moment-tomass ratios of $\mathrm{Rb}$ and $\mathrm{Cs}$.

The mixture is held in the optical trap for $2 \mathrm{~s}$ to allow for thermalization and plain evaporation. In single-species experiments, $\mathrm{Rb}$ atoms have a lifetime of about $10 \mathrm{~s}$ in the dipole trap. A shorter lifetime of about $3 \mathrm{~s}$ is measured for $\mathrm{Cs}$ atoms [38].

We observe that $\mathrm{Cs}$ and $\mathrm{Rb}$ atoms rapidly thermalize during the evaporation via sympathetic cooling, which points to a large cross section for interspecies elastic collisions. Because of the different trap depths, the evaporative cooling preferentially leads to losses of $\mathrm{Rb}$ atoms. To produce a mixture with equal atom number, we start with a Cs MOT that has only $10^{7}$ atoms. In the dipole trap we then finally measure $6 \times 10^{5}$ atoms for each species at a temperature of about $3 \mu \mathrm{K}$ and peak density of a few $10^{10} \mathrm{~cm}^{-3}$. By further reducing the MOT loading, we can also produce an imbalanced mixture with much more $\mathrm{Rb}$ than $\mathrm{Cs}$ atoms. Note that for the present application of Feshbach spectroscopy it was not necessary to optimize the mode matching between the Raman cooled clouds and the dipole trap, resulting in a low loading efficiency of the trap [39].

For Feshbach spectroscopy experiments, it is favorable to increase the atomic density of both species since the losses occur as a result of interspecies collisions. We compress the mixture by superimposing an additional tightly focused laser beam, which acts as a "dimple trap" [15, 16]. The optical field is generated by a second fiber laser with central wavelength of about $1064 \mathrm{~nm}$ and linewidth of about $1 \mathrm{~nm}$. The beam has a power of about $300 \mathrm{~mW}$ and a waist of $38 \mu \mathrm{m}$. The dimple trap depths are $18 \mu \mathrm{K}$ and $31 \mu \mathrm{K}$ for $\mathrm{Rb}$ and $\mathrm{Cs}$, respectively. With the use of this additional optical field, we typically increase the peak density of both species by an order of magnitude to about $5 \times 10^{11} \mathrm{~cm}^{-3}$ at a final temperature of about $7 \mu \mathrm{K}$.

\section{Rb-Cs FESHBACH RESONANCES}

A Feshbach resonance manifests itself in an enhancement of trap loss [17]. In the energetically lowest spin channel, such losses can be fully attributed to three-body collisions. In our mixture, three-body collisions involving one Cs and two $\mathrm{Rb}$ atoms and collisions with one $\mathrm{Rb}$ and two $\mathrm{Cs}$ atoms are both possible. The rate coefficients for these two processes can in general be different, with one process dominating over the other. The visibility of heteronuclear resonances can be enhanced by creating an imbalance between the atom numbers of the two species [4, 40]. In this way, one species can be used as a probe and the other one as a collisional bath. For large enough atom imbalance, the minority component will be fully depleted, while the majority component will remain nearly unaffected. By a selective control of the MOT loading, we create a majority species (either $\mathrm{Rb}$ or $\mathrm{Cs}$ ) and a minority species (thus either $\mathrm{Cs}$ or $\mathrm{Rb}$ ) with an imbalance ratio of about $1: 10$.

\section{A. Energetically lowest spin channel}

We perform Feshbach spectroscopy in the absolute ground state of the two species, i. e. in their lowest spin-state combination $\mathrm{Rb}|1,1\rangle+\mathrm{Cs}|3,3\rangle$. We search for heteronuclear resonances by performing a magnetic field scan between 20 and $300 \mathrm{G}$ with a step size of $250 \mathrm{mG}$. At low magnetic fields our scan is limited by the horizontal forces induced by the magnetic levitation field, which pull the atoms out of the trap [15]. A scan consists of more than 1000 experimental cycles of about $30 \mathrm{~s}$ duration each. In each cycle, we let the mixture evolve at a specific value of the homogeneous magnetic field for $5 \mathrm{~s}$. In the next cycle, we change the magnetic field value in random order and we repeat the measurement. To determine the atom number of the minority species, we switch off the optical dipole trap, recapture the remaining atoms into the MOT, and record their fluorescence signal. We trace the full loss spectrum using $\mathrm{Cs}$ atoms as probe; for some resonances 


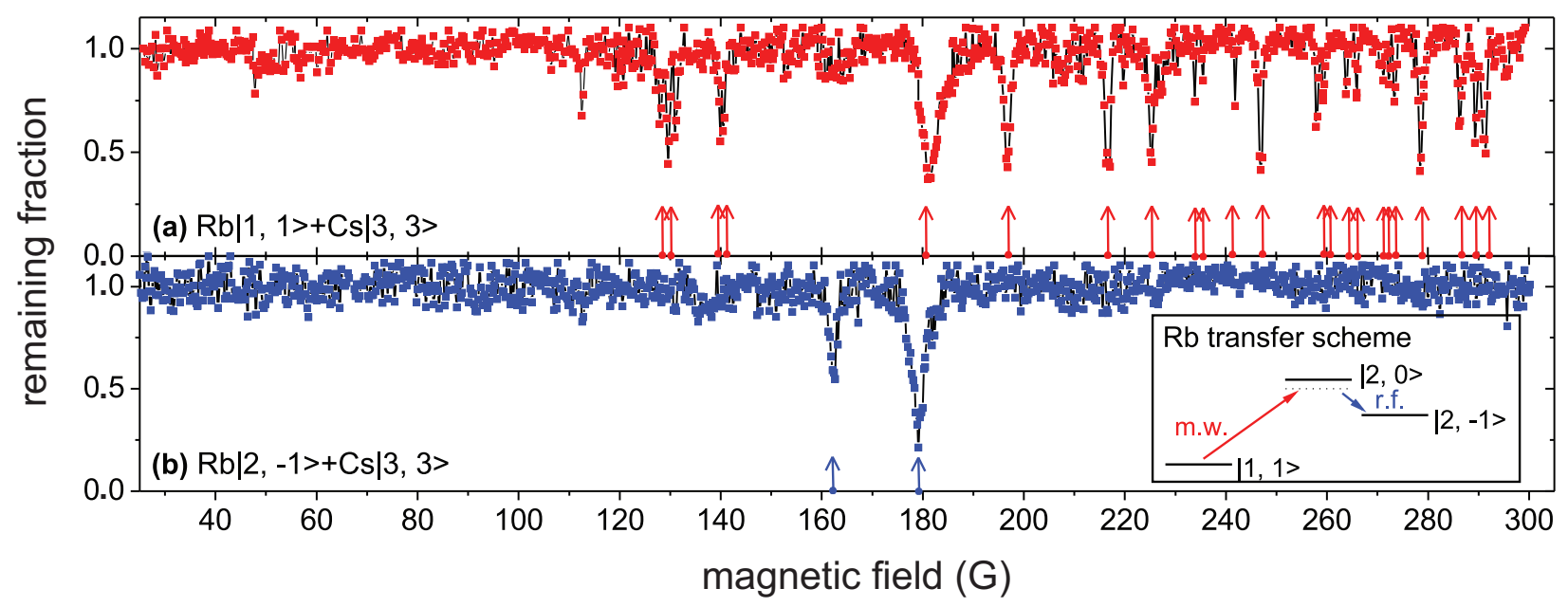

FIG. 2: color online. Observation of heteronuclear Rb-Cs Feshbach resonances by loss spectroscopy. The spectrum is recorded for Cs atoms, being the minority component of the mixture. The remaining fraction represents the number of Cs atoms after a 5-s hold time in the dipole trap normalized to the corresponding average number detected off any resonance. The arrows indicate the positions of the interspecies Feshbach resonances. Other loss features correspond to homonuclear Cs Feshbach resonances. (a) shows the loss spectrum for the Rb $|1,1\rangle+\mathrm{Cs}|3,3\rangle$ channel. (b) displays the $\mathrm{Rb}|2,-1\rangle+\mathrm{Cs}|3,3\rangle$ channel. The inset illustrates the transfer scheme applied to populate the $\mathrm{Rb}|2,-1\rangle$ state.

we also perform consistency checks by monitoring the loss of $\mathrm{Rb}$ atoms prepared as a minority species.

Figure 2 a) shows the complete loss spectrum choosing Cs as the probe. Each point corresponds to an average of 5 to 10 different measurements. At specific values of the magnetic field, the Cs atom number decreases by at least $20 \%$. Since the mixture contains an order of magnitude less $\mathrm{Cs}$ than $\mathrm{Rb}$ atoms, the latter species exhibits very small relative losses. To avoid any possible confusion with homonuclear resonances, we have repeated the magnetic field scan using samples of either pure $\mathrm{Cs}$ or pure $\mathrm{Rb}$ atoms. The loss features, indicated by the arrows in Fig.2(a), only appear using a two-species mixture, while the others can be attributed to Cs Feshbach resonances [18].

In this way, we identify 23 interspecies $\mathrm{Rb}-\mathrm{Cs}$ Feshbach resonances in the absolute ground state combination of the two species.

Two main mechanisms can contribute to a broadening of the loss resonances. First, the levitation field gives rise to an inhomogeneous broadening, which we estimate to be of about $70 \mathrm{mG}$. Second, the finite temperature of the mixture leads to a broadening on the order of $100 \mathrm{mG}$ [41]. As a result of both broadening contributions, our resolution limit is not better than $100 \mathrm{mG}$. Therefore we extract widths from our experimental data only for those resonances that appear wider than this resolution limit. To determine the full widths at half maximum, $\Delta B$, we use Lorentzian fits. The measured positions, widths, and loss fractions are summarized in Table [

\section{B. Higher spin channel}

In a second set of experiments, we perform Feshbach spectroscopy in a higher spin channel. We keep the Cs atoms in the absolute ground state because of strong two-body dipo-
TABLE I: Feshbach resonances in collisions between ${ }^{87} \mathrm{Rb}$ and ${ }^{133} \mathrm{Cs}$ in a range from 20 to $300 \mathrm{G}$. The locations, the widths $\Delta B$ and the fractional losses of the Feshbach resonances in both channels $\mathrm{Rb}|1,1\rangle+\mathrm{Cs}|3,3\rangle$ and $\mathrm{Rb}|2,-1\rangle+\mathrm{Cs}|3,3\rangle$ are listed.

\begin{tabular}{c|cccccc}
\hline \hline & $\mathrm{B}(\mathrm{G})$ & loss $(\%)$ & $\Delta B(\mathrm{G})$ & $\mathrm{B}(\mathrm{G})$ & loss $(\%)$ & $\Delta B(\mathrm{G})$ \\
\hline $\mathrm{Rb}|1,1\rangle$ & 128.0 & 40 & - & 258.0 & 55 & 0.4 \\
$+\mathrm{Cs}|3,3\rangle$ & 129.6 & 60 & - & 259.3 & 30 & 0.5 \\
& 140.0 & 50 & - & 263.8 & 30 & 0.5 \\
& 140.5 & 50 & - & 265.9 & 30 & - \\
& 181.6 & 70 & 3.1 & 271.2 & 25 & - \\
& 196.8 & 60 & 1.2 & 272.3 & 25 & 0.3 \\
& 216.7 & 60 & 1 & 273.4 & 30 & 0.4 \\
& 225.3 & 60 & 1 & 278.4 & 65 & 0.95 \\
& 233.9 & 30 & - & 286.2 & 45 & 0.8 \\
& 235.5 & 25 & - & 289.4 & 50 & 0.5 \\
& 241.9 & 35 & - & 291.5 & 55 & 1.1 \\
& 246.9 & 65 & 0.7 & & & \\
$\mathrm{Rb}|2,-1\rangle$ & 162.3 & 50 & 1.4 & & & \\
$+\mathrm{Cs}|3,3\rangle$ & 179.1 & 80 & 2.8 & & & \\
\hline \hline
\end{tabular}

lar losses occurring for Cs-Cs collisions in any higher spin state [42]. The $\mathrm{Rb}|2,-1\rangle$ state is the only other state simultaneously fulfilling the levitation condition. Atoms in the $\mathrm{Rb}|2,-1\rangle+\mathrm{Cs}|3,3\rangle$ channel are stable against spin-exchange collisions that do not involve a change in $F$ as the relaxation channels are energetically closed. Two-body hyperfinechanging collisions are energetically possible and allowed under the conservation of the total orientation quantum number, but we do not observe any significant background loss that exceeds the observations in the other spin channel. Loss at Fesh- 
bach resonances may however include resonantly enhanced two-body loss.

We start with $\mathrm{Rb}$ atoms prepared in the $\mathrm{Rb}|1,1\rangle$ state as described before. Then we apply a two-photon transfer scheme, which combines microwave and radio-frequency excitation. The inset of Fig.2 (b) schematically shows the working principle of the transfer scheme. The microwave frequency is detuned by few $\mathrm{MHz}$ with respect to the $\mathrm{Rb}|1,1\rangle \rightarrow \mathrm{Rb}|2,0\rangle$ transition. The radio-frequency signal couples the $\mathrm{Rb}|2,0\rangle$ and the $\mathrm{Rb}|2,-1\rangle$ state with the corresponding detuning that leads to the two-photon resonance [43].

The pulse sequence has a duration of $400 \mathrm{~ms}$ during which the magnetic field value is set at $20 \mathrm{G}$. Using this scheme, we incoherently transfer almost $50 \%$ of the ground state $\mathrm{Rb}$ atoms into the excited state [44]. The remaining atoms in the $\mathrm{Rb}|1,1\rangle$ state are selectively removed from the trap by applying a short light pulse resonant with the $F=1 \rightarrow F^{\prime}=2$ transition, pumping the atoms into different magnetic sublevels of the $F=2$ upper hyperfine state. Those atoms which do not end up in the target state $\mathrm{Rb}|2,-1\rangle$ leave the trap as the levitation condition is not fulfilled. In this way spin impurities can be completely avoided.

To probe Feshbach resonances, we again perform a magnetic field scan, as previously described. As shown in Fig.2[b), we observe two resonant loss features. The positions, the widths, and the loss fractions are listed in Table $\square$ The observation of just two resonances in this higher spin channel stands in contrast to the multitude of resonances observed in the energetically lowest channel.

\section{DISCUSSION}

The interspecies scattering behavior of the ultracold $\mathrm{Rb}-\mathrm{Cs}$ system is still an open issue. The only piece of information available prior to the present work stems from experiments in magnetically trapped $\mathrm{Rb}$-Cs mixtures [20, 21]. The fast thermalization observed between $\mathrm{Rb}$ and $\mathrm{Cs}$, both being in their doubly-polarized states, points to a large elastic cross section. A theoretical analysis [23] has identified two possible values of the interspecies triplet scattering length, $700_{-300}^{+700} a_{0}$ and $176_{-2}^{+2} a_{0}$, the first value being the more likely one [23]. Information on the scattering behavior associated with the singlet potential and on the near-threshold molecular structure is totally absent. Some knowledge on deeply bound states is available from photoassociation experiments, in which a sample of $\mathrm{RbCs}$ molecules was produced in the rovibrational ground state [45].

Our Feshbach spectroscopy opens up a way to substantially improve our understanding of the interspecies scattering properties and the near-threshold molecular structure of the RbCs system. The resonance positions provide valuable experimental input to determine the relevant parameters of quantum collisional models, such as the asymptotic bound state model [40, 46] and coupled channels calculations [47]. Such scattering models have been successfully applied to the case of lighter combinations, e. g. the $\mathrm{Li}-\mathrm{K}$ [40] and the $\mathrm{Rb}-\mathrm{K}$ [48, 49] mixture. However, the resonance assignment for heavier com- binations, such as the Rb-Cs system, is much more challenging because of a larger hyperfine energy, a smaller vibrational splitting in the molecular potentials, the spin-spin interactions (second order spin-orbit and magnetic dipole interaction), and odd partial-wave contributions to the scattering of non-identical bosons. These effects result in an increase of the density of the relevant bound states and in a mixing of different molecular levels.

The Feshbach spectrum observed for the Rb-Cs system reflects a variety of near-threshold molecular states and appears to be much richer than the spectra observed for lighter mixtures [4, 40, 46, 48, 50]. In the Rb-Cs combination, the total hyperfine energy of about $h \times 16 \mathrm{GHz}$ substantially exceeds the near-threshold vibrational splitting, resulting in a mixing of at least five vibrational levels [51]. A large second-order spin-orbit interaction, which mixes states with different angular momenta $\ell$ up to $\Delta \ell=4$, is likely. In particular, $\mathrm{Rb}$ and $\mathrm{Cs}$ atoms colliding in an $s$ wave may couple to $s-, d$-, and $g$-wave $\mathrm{RbCs}$ molecular states via Feshbach resonances. The important role of higher-order coupling effects is well known from ultracold interactions in cesium samples [52]. It has, for example, been demonstrated by populating molecular states with high orbital angular momentum [53, 54, 55]. In a mixture, collisions between non-identical particles can also take place in odd partial waves. In our temperature regime $(T=7 \mu \mathrm{K})$, $p$-wave collisions may be particularly important as the height of the corresponding centrifugal potential barrier is only about $k_{\mathrm{B}} \times 55 \mu \mathrm{K}$. The $p$-wave resonances can be induced by $p$-wave molecular states, or also through higher-order couplings by $f$ wave or $h$-wave states.

A $p$-wave resonance can lead to a doublet structure because of the spin-spin dipole interaction, which splits the resonance into two components based on the partial-wave projection onto the magnetic field axis, $m_{\ell}=0$ and $\left|m_{\ell}\right|=1$ [56]. In our Feshbach scan, we observe several resonances that come in pairs. Such a feature could be fortuitous but could also be the evidence of the $p$-wave character. In particular, four resonance pairs are candidates for $p$-wave resonances, namely the resonances near 140, 234, 259, and $273 \mathrm{G}$. We typically observe a splitting of about $1 \mathrm{G}$, which would indicate a strong spin-spin interaction between $\mathrm{Rb}$ and $\mathrm{Cs}$ atoms. Doublets with a splitting of this order have been predicted for the specific $\mathrm{Rb}-\mathrm{Cs}$ system [57]. Figure 3 gives an example of such a doublet structure. Here, the splitting between the two peaks is about $1.3 \mathrm{G}$.

First calculations, based both on the asymptotic bound state model and coupled channels theory, confirm the extraordinary richness of the Rb-Cs spectrum [51, 57]. Coupled channel calculations also show the importance of second-order spin-orbit and spin-spin interaction and predict doublet structures of $p$ wave states [57]. However, further refinements of the models are needed to reach an assignment of the observed resonances.

\section{OUTLOOK}

The observation of a variety of Feshbach resonances opens up several intriguing avenues of research both with tunable 


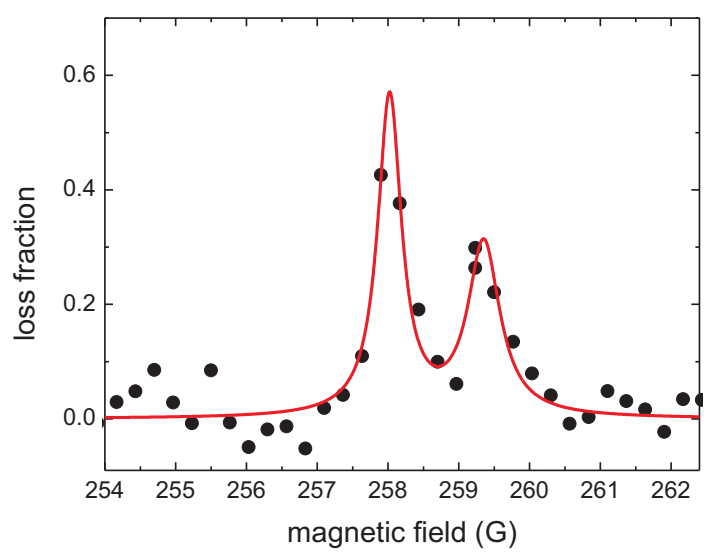

FIG. 3: color online. Example of a Rb-Cs resonance pair observed in the $\mathrm{Rb}|1,1\rangle+\mathrm{Cs}|3,3\rangle$ channel. The circles represents a subset of the data shown in Fig.2 and the solid line is a fit to the data using a double Lorentzian profile. The resonance splitting is about $1.3 \mathrm{G}$.

mixtures and heteronuclear molecules. The production of a tunable double BEC will allow to access complex quantum phenomena, including phase separation, selective collapse, and vortex formation [58, 59, 60, 61]. With the additional use of an optical lattice, the superfluid-to-Mott insulator transition $[62,63,64]$ and repulsively bound pairs with an imbalance of masses [65] can be studied. Heteronuclear resonances are also relevant in few-body physics because of the possible enhancement of the observability of the Efimov effect [66, 67].

On the molecular side, the observed Feshbach resonances can be used as an entrance gate for association of molecules of different character. We expect that RbCs molecules can be, for instance, associated by ramping the magnetic field value across any of the narrow and broad interspecies Feshbach resonances [68, 69]. Two particularly interesting directions can be pursued with heteronuclear molecules: universal physics with complex few-body structures and dipolar gases. The comparatively broad resonance located at about $180 \mathrm{G}$ may be a candidate for an entrance-channel dominated resonance [17, 70]. Such a resonance could serve for the production of weakly bound molecules of halo character in a regime of large scattering length [17, 71] and to address Efimov states composed of unequal mass particles [72].

Dipolar gases of heteronuclear molecules in the rovibrational ground state have recently attracted widespread attention because of the potentially strong intermolecular dipolar force, which shows a long-range and anisotropic character $[12,73,74]$. As recently demonstrated, such a system can be produced by first associating Feshbach molecules and by then applying a coherent transfer scheme to populate the rovibrational ground state [12, 26, 27, 75]. Theoretical work [24] has predicted strong dipolar forces between $\mathrm{RbCs}$ molecules in the rovibrational ground state.

\section{Acknowledgments}

We thank S. Kokkelmans, S. Kotochigova, M. Goosen, and E. Tiesinga for stimulating discussions and for sharing their insights in the Rb-Cs system. We acknowledge support by the Austrian Science Fund (FWF) within SFB 15 (project part 16) and by the European Science Foundation (ESF) in the framework of the EuroQUAM collective research project QuDipMol. F. F. is supported within the Lise Meitner program of the FWF.
[1] G. Modugno, G. Ferrari, G. Roati, R. J. Brecha, A. Simoni, and M. Inguscio, Science 294, 1320 (2001).

[2] Z. Hadzibabic, C. A. Stan, K. Dieckmann, S. Gupta, M. W. Zwierlein, A. Görlitz, and W. Ketterle, Phys. Rev. Lett. 88, 160401 (2002).

[3] G. Roati, F. Riboli, G. Modugno, and M. Inguscio, Phys. Rev. Lett. 89, 150403 (2002).

[4] B. Deh, C. Marzok, C. Zimmermann, and P. W. Courteille, Phys. Rev. A 77, 010701(R) (2008).

[5] M. Taglieber, A.-C. Voigt, T. Aoki, T. W. Hänsch, and K. Dieckmann, Phys. Rev. Lett. 100, 010401 (2008).

[6] F. Schreck, L. Khaykovich, K. L. Corwin, G. Ferrari, T. Bourdel, J. Cubizolles, and C. Salomon, Phys. Rev. Lett. 87, 080403 (2001).

[7] K. Honda, Y. Takasu, T. Kuwamoto, M. Kumakura, Y. Takahashi, and T. Yabuzaki, Phys. Rev. A 66, 021401 (2002).

[8] S. B. Papp and C. E. Wieman, Phys. Rev. Lett. 97, 180404 (2006).

[9] J. M. McNamara, T. Jeltes, A. S. Tychkov, W. Hogervorst, and W. Vassen, Phys. Rev. Lett. 97, 080404 (2006).

[10] C. Ospelkaus, S. Ospelkaus, L. Humbert, P. Ernst, K. Sengstock, and K. Bongs, Phys. Rev. Lett. 97, 120402 (2006).

[11] C. Weber, G. Barontini, J. Catani, G. Thalhammer, M. Inguscio, and F. Minardi, arXiv:0808.4077 (2008).
[12] K.-K. Ni, S. Ospelkaus, M. H. G. de Miranda, A. Pe'er, B. Neyenhuis, J. J. Zirbel, S. Kotochigova, P. S. Julienne, D. S. Jin, and J. Ye, Science 322, 231 (2008).

[13] M. Baranov, Phys. Rep. 464, 71 (2008).

[14] M. H. Anderson, J. R. Ensher, M. R. Matthews, C. E. Wieman, and E. A. Cornell, Science 269, 198 (1995).

[15] T. Weber, J. Herbig, M. Mark, H.-C. Nägerl, and R. Grimm, Science 299, 232 (2003).

[16] T. Kraemer, J. Herbig, M. Mark, T. Weber, C. Chin, H.-C. Nägerl, and R. Grimm, Appl. Phys. B 79, 1013 (2004).

[17] C. Chin, R. Grimm, P. S. Julienne, and E. Tiesinga, arXiv:0812.1496 (2008).

[18] C. Chin, V. Vuletić, A. J. Kerman, S. Chu, E. Tiesinga, P. J. Leo, and C. J. Williams, Phys. Rev. A 70, 032701 (2004).

[19] A. Marte, T. Volz, J. Schuster, S. Dürr, G. Rempe, E. G. M. van Kempen, and B. J. Verhaar, Phys. Rev. Lett. 89, 283202 (2002).

[20] M. Anderlini, E. Courtade, M. Cristiani, D. Cossart, D. Ciampini, C. Sias, O. Morsch, and E. Arimondo, Phys. Rev. A. 71, 061401(R) (2005).

[21] M. Haas, V. Leung, D. Frese, D. Haubrich, S. John, C. Weber, A. Rauschenbeutel, and D. Meschede, New J. Phys. 9, 147 (2007).

[22] M. Harris, P. Tierney, and S. L. Cornish, J. Phys. B. 41, 035303 (2008). 
[23] E. Tiesinga, M. Anderlini, and E. Arimondo, Phys. Rev. A. 75, 022707 (2007).

[24] S. Kotochigova and E. Tiesinga, J. Chem. Phys. 123, 174304 (2005).

[25] F. Ferlaino, S. Knoop, and R. Grimm, in Cold Molecules: Theory, Experiment, Applications, edited by R. V. Krems, B. Friedrich, and W. C. Stwalley (2008), arXiv:0809.3920.

[26] J. G. Danzl, E. Haller, M. Gustavsson, M. J. Mark, R. Hart, N. Bouloufa, O. Dulieu, H. Ritsch, and H.-C. Nagerl, Science 321, 1062 (2008).

[27] F. Lang, K. Winkler, C. Strauss, R. Grimm, and J. Hecker Denschlag, Phys. Rev. Lett. 101, 133005 (2008).

[28] K. Pilch, PhD thesis, Univ. Innsbruck (2009).

[29] A. J. Kerman, V. Vuletić, C. Chin, and S. Chu, Phys. Rev. Lett. 84, 439 (2000).

[30] P. Treutlein, K. Y. Chung, and S. Chu, Phys. Rev. A 63, 051401 (2001).

[31] M.Weidemüller, M. Reetz-Lamour, T. Amthor, J. Deiglmayr, and K. Singer, Laser Spectroscopy XVII, edited by E.A. Hinds, A. Ferguson, and E. Riis (World Scientific, New Jersey, 2005) p. 264 (2005).

[32] G. Grynberg, B. Lounis, P. Verkerk, J. Y. Courtois, and C. Salomon, Phys. Rev. Lett. 70, 2249 (1993).

[33] This light is resonant with the $6 S_{1 / 2}(F=4) \rightarrow 6 P_{3 / 2}\left(F^{\prime}=4\right)$ transition and simultaneously serves to form the lattice and to depopulate the $F=4$ state [16].

[34] D. J. Han, M. T. DePue, and D. S. Weiss, Phys. Rev. A 63, 023405 (2001).

[35] M. Gustavsson, E. Haller, M. J. Mark, J. G. Danzl, G. RojasKopeinig, and H.-C. Nägerl, Phys. Rev. Lett. 100, 080404 (2008).

[36] C.-L. Hung, X. Zhang, N. Gemelke, and C. Chin, Phys. Rev. A 78, 011604 (2008).

[37] The crossing angle is about $78^{\circ}$ for reason of optical access to the trapping cell.

[38] We believe the shorter lifetime of Cs atoms to be due to the $6 s \rightarrow 7 s$ two-photon transition driven by the dipole trap light.

[39] In later experiments with optimized loading [28] we could demonstrate BEC of $\mathrm{Cs}$ in this setup.

[40] E. Wille, F. M. Spiegelhalder, G. Kerner, D. Naik, A. Trenkwalder, G. Hendl, F. Schreck, R. Grimm, T. G. Tiecke, J. T. M. Walraven, S. J. J. M. F. Kokkelmans, E. Tiesinga, and P. S. Julienne, Phys. Rev. Lett. 100, 053201 (2008).

[41] For a typical cloud size of $\sigma=20 \mu \mathrm{m}$, we estimate the broadening caused by the field gradient $B^{\prime}$ to be $B^{\prime} \sigma \approx 70 \mathrm{mG}$. The temperature broadening can be estimated as $k_{\mathrm{B}} T / \Delta \mu$, where $\Delta \mu$ is the relative magnetic moment between the atom pair state and the molecular state causing the resonance. The broadening typically varies between 50 and $200 \mathrm{mG}$.

[42] D. Guéry-Odelin, J. Söding, P. Desbiolles, and J. Dalibard, Europhys. Lett. 44, 25 (1998).

[43] We choose this two-photon transfer scheme because it is insensitive to magnetic field fluctuations, whereas two subsequent one-photon transitions would be strongly affected.

[44] A coherent transfer of Rb atoms could not be successfully implemented our setup. We believe the magnetic gradient field in combination with the residual magnetic-field dependence of the two-photon transition to be the main reason.

[45] J. M. Sage, S. Sainis, T. Bergeman, and D. DeMille, Phys. Rev. Lett. 94, 203001 (2005).
[46] C. A. Stan, M. W. Zwierlein, C. H. Schunck, S. M. F. Raupach, and W. Ketterle, Phys. Rev. Lett. 93, 143001 (2004).

[47] H. T. C. Stoof, J. M. V. A. Koelman, and B. J. Verhaar, Phys. Rev. B 38, 4688 (1988).

[48] F. Ferlaino, C. D’Errico, G. Roati, M. Zaccanti, M. Inguscio, G. Modugno, and A. Simoni, Phys. Rev. A 73, 040702 (2006).

[49] C. Klempt, T. Henninger, O. Topic, J. Will, W. Ertmer, E. Tiemann, and J. Arlt, Phys. Rev. A 76, 020701 (2007).

[50] S. Inouye, J. Goldwin, M. L. Olsen, C. Ticknor, J. L. Bohn, and D. S. Jin, Phys. Rev. Lett. 93, 183201 (2004).

[51] S. J. J. M. F. Kokkelmans, private communication (2008).

[52] C. Chin, V. Vuletić, A. J. Kerman, and S. Chu, Phys. Rev. Lett. 85, 2717 (2000).

[53] M. Mark, F. Ferlaino, S. Knoop, J. G. Danzl, T. Kraemer, C. Chin, H.-C. Nägerl, and R. Grimm, Phys. Rev. A 76, 042514 (2007).

[54] M. Mark, T. Kraemer, P. Waldburger, J. Herbig, C. Chin, H.-C. Nägerl, and R. Grimm, Phys. Rev. Lett. 99, 113201 (2007).

[55] S. Knoop, M. Mark, F. Ferlaino, J. G. Danzl, T. Kraemer, H.-C. Nägerl, and R. Grimm, Phys. Rev. Lett. 100, 083002 (2008).

[56] C. Ticknor, C. A. Regal, D. S. Jin, and J. L. Bohn, Phys. Rev. A 69, 042712 (2004).

[57] S. Kotochigova, private communication (2008).

[58] J. L. Roberts, N. R. Claussen, S. L. Cornish, E. A. Donley, E. A. Cornell, and C. E. Wieman, Phys. Rev. Lett. 86, 4211 (2001).

[59] E. Timmermans, Phys. Rev. Lett. 81, 5718 (1998).

[60] J. J. García-Ripoll and V. M. Pérez-García, Phys. Rev. Lett. 84, 4264 (2000).

[61] M. Zaccanti, C. D’Errico, F. Ferlaino, G. Roati, M. Inguscio, and G. Modugno, Phys. Rev. A 74, 041605 (2006).

[62] M. Lewenstein, L. Santos, M. A. Baranov, and H. Fehrmann, Phys. Rev. Lett. 92, 050401 (2004).

[63] G. Thalhammer, G. Barontini, L. De Sarlo, J. Catani, F. Minardi, and M. Inguscio, Phys. Rev. Lett. 100, 210402 (2008).

[64] I. Bloch, J. Dalibard, and W. Zwerger, Rev. Mod. Phys. 80, 885 (2008).

[65] K. Winkler, G. Thalhammer, F. Lang, R. Grimm, J. Hecker Denschlag, A. J. Daley, A. Kantian, H. P. Büchler, and P. Zoller, Nature 441, 853 (2006).

[66] J. P. D’Incao and B. D. Esry, Phys. Rev. A 73, 030703 (2006).

[67] T. Kraemer, M. Mark, P. Waldburger, J. G. Danzl, C. Chin, B. Engeser, A. D. Lange, K. Pilch, A. Jaakkola, H.-C. Nägerl, and R. Grimm, Nature 440, 315 (2006).

[68] J. Herbig, T. Kraemer, M. Mark, T. Weber, C. Chin, H.-C. Nägerl, and R. Grimm, Science 301, 1510 (2003).

[69] C. A. Regal, C. Ticknor, J. L. Bohn, and D. S. Jin, Nature 424, 47 (2003).

[70] T. Köhler, K. Góral, and P. S. Julienne, Rev. Mod. Phys. 78, 1311 (2006).

[71] F. Ferlaino, S. Knoop, M. Mark, M. Berninger, H. Schöbel, H.C. Nägerl, and R. Grimm, Phys. Rev. Lett. 101, 023201 (2008).

[72] E. Braaten and H.-W. Hammer, Phys. Rep. 428, 259 (2006).

[73] K. Góral, L. Santos, and M. Lewenstein, Phys. Rev. Lett. 88, 170406 (2002).

[74] S. Yi, T. Li, and C. P. Sun, Phys. Rev. Lett. 98, 260405 (2007).

[75] K. Winkler, F. Lang, G. Thalhammer, P. van der Straten, R. Grimm, and J. Hecker Denschlag, Phys. Rev. Lett. 98, 043201 (2007). 